\title{
Utility Locating in the DOE Environment
}

\section{SAGEEP 2006}

\author{
Clark Scott \\ Gail Heath
}

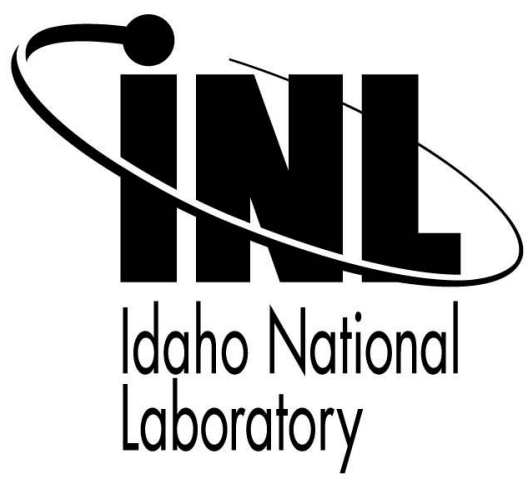

This is a preprint of a paper intended for publication in a journal or proceedings. Since changes may be made before publication, this preprint should not be cited or reproduced without permission of the author. This document was prepared as an account of work sponsored by an agency of the United States Government. Neither the United States Government nor any agency thereof, or any of their employees, makes any warranty, expressed or implied, or assumes any legal liability or responsibility for any third party's use, or the results of such use, of any information, apparatus, product or process disclosed in this report, or represents that its use by such third party would not infringe privately owned rights. The views expressed in this paper are not necessarily those of the United States Government or the sponsoring agency. 


\title{
UTILITY LOCATING IN THE DOE ENVIRONMENT
}

\author{
Clark Scott, INL, Idaho
}

Gail Heath, INL, Idaho

\begin{abstract}
Some advances have been made in utility locating in recent years and standards have been recently published to try and categorize the level of information known about the utilities in the subsurface. What current standards lack is characterization about the level of effort or technology in the geophysicist approach to utility locating. A simple generalization of geophysical technology/product, designed to fit within current standards, is advanced for the purpose of discussion. This generalization uses three levels or tiers to distinguish the geophysical product and its inherent information content. Due to nuclear legacy issues, the Department of Energy (DOE) environment poses some added difficulties and this generalization is used to describe the technical approach, with examples, that is being developed at the Idaho National Laboratory.
\end{abstract}

\section{Introduction}

Recent discoveries of unanticipated buried utility lines at The Idaho National Laboratory (INL), in areas where utility-locating surveys had just been completed, point to the need for improving utility locating technologies. This problem is not new nor is it unique to the INL. Outside the costly DOE work environment at the INL (\$50k per hit), costs and disruptions caused by damage to in-place utilities can run into the millions of dollars. A recent report on utility locating distributed by the Federal Laboratory Consortium for Technology Transfer (Sterling, 2000) states "Individual incidents can entail costs that are out of proportion with the cost of the work being undertaken, and the total of all utility damage costs is very significant and increasing".

The problems being encountered at the INL and elsewhere point to a need for improvements in locating techniques. However the subject of utility locating covers a broad category of procedures and technologies that span the range from historical archive searches to geophysical prospecting to information technology issues such as integrated database and Geographical Information Systems (GIS). The Sterling report mentioned above was written to summarize responses to the following statement of need (SON) originally put out by the Federal Laboratory Consortium for Technology Transfer.

What is desired is a single multisensor system that accurately locates all underground utilities under the variety of site conditions found in urban areas. Ideally, the method(s) would operate from the ground surface and not require prior knowledge of the location of or access to the utility to introduce special signals for detection. Novel approaches, sensors, and/or a combination of technologies are needed to increase the reliability of utility detection in terms of the size, depth, and utility materials that can be detected and operate in the presence of utility congestion and error-producing conditions present in urban rights-of-way.

The SON is rather focused on the geophysical aspects of locating but is a good example of the level of technology being sought throughout the continuum of utility locating. The report details the responses and reflects a comprehensive but not complete spectrum of current 'state of the art' 
technologies. The conclusion from this effort can be summed up by the following questions/statements taken from the report.

- Were any relevant technologies uncovered that were not currently being applied to the utility locating problem?

The responses did not indicate any relevant technologies that had not been considered for application to the utility location problem.

- Is there a system under current development that will be capable of locating urban utilities as desired in the Statement of Need

None of the identified technologies is capable of providing a complete solution to the utility location problem.

More importantly, the results indicated that while there was little to report on new geophysical technologies, there is a lot of progress to be made in enhancing utility locating with the existing technologies by improving data collection, integration and information management. Finally the underlying problems at the INL and at other sites are succinctly stated in the following response.

- What else is needed in the industry to lower utility damage?

Many existing problems dealing with utility damage are organizational in nature.

Here organizational really means information management and communication. One aspect of this organizational need is the requirement for standards in the industry.

\section{Utility Locating Standards}

Historically the utility locating industry has been composed of diverse small service companies with little or no external standards for the work they do. Some of the larger companies, such as SO$\mathrm{DEEP}^{1}$ inc., have been addressing this problem and have attempted to standardize and define terminology and standards, within their own sphere of influence, with respect to the wider subject of utility locating commonly referred to now as Subsurface Utility Engineering (SUE). These companies have been driving the acceptance of common definitions such as "designating' (interpreting utility presence from geophysics) vs. "locating" (Exposing and recording actual utility position) and introduced a scale for defining quality levels within utility locating.

Recently the American Society of Civil Engineers (ASCE) has published a set of standards that has embraced the terminology developed by SO-DEEP and others (ASCE,2003), as well as specifying the scope of responsibility for the parties concerned with the utility investigation. Within this standard are four recognized levels of quality of knowledge concerning actual utility locations.

\section{${ }^{1}$ PRODUCT DISCLAIMER}

References herein to any specific commercial product, process, or service by trade name, trademark, manufacturer, or otherwise, does not necessarily constitute or imply its endorsement, recommendation, or favoring by the U.S. Government, any agency thereof, or any company affiliated with the Idaho National Laboratory. 
Table 1: ASCE quality levels from lowest to highest

\begin{tabular}{|c|l|}
\hline Quality & \multicolumn{1}{c|}{ Description } \\
\hline D & Information derived from existing records or oral recollections \\
\hline C & $\begin{array}{l}\text { Information obtained by surveying and plotting visible above-ground utility features and } \\
\text { by using professional judgment in correlating this information to quality level D } \\
\text { Information. }\end{array}$ \\
\hline B & $\begin{array}{l}\text { Information obtained through the application of appropriate surface geophysical } \\
\text { methods to determine the existence and approximate horizontal position of subsurface } \\
\text { utilities. This information is surveyed to applicable tolerances defined by the project and } \\
\text { reduced onto plan documents. }\end{array}$ \\
\hline A & $\begin{array}{l}\text { Precise horizontal and vertical location of utilities obtained by the actual exposure and } \\
\text { subsequent measurement of subsurface utilities, usually at a specific point. A precise } \\
\text { horizontal and vertical location, as well as other utility attributes, is shown on plan } \\
\text { documents. Accuracy is typically set to 15-mm vertical and to applicable horizontal } \\
\text { survey and mapping accuracy as defined or expected by the project owner. }\end{array}$ \\
\hline
\end{tabular}

Since the publication of the standards governmental organizations responsible for authorizing utility and excavation work are beginning to require at minimum a quality level B prior to excavation. This is also the level that we as geophysicist are most concerned with as it represents our main contribution to the utility locating process. At this level, utility designation is essentially a process involving shallow geophysical surveying, data management and depiction. However within the ASCE published standards there is no clear description of what level of geophysics is required to satisfy level B requirements. In practice the typical geophysical product can range from paint-on-the-ground in a simple radio frequency locating effort to the migrated and geo-referenced mapped product supplied by the Witten Technologies cart imaging system.

In an effort to better describe, and hence better sell, the geophysical aspect of utility locating, work at the INL has been ongoing to broadly classify the available geophysical product levels that fit within the ASCE quality level B description. This generalization makes use of three levels or tiers to describe the geophysical product and its information content. These concepts are shown in examples that represent the increasing expectations at the INL.

\section{Geophysical Product Tiers}

For purpose of discussion the level of technology or 'product' supplied by the geophysical industry can be divided into three tiers (Figure 1). Unlike the standards based terminology for quality described above, this ranking is still local to the INL and is not currently found in the industry. Unfortunately most efforts at the INL and elsewhere have historically fallen in the lowest of these tiers and the less than optimal results have contributed, within the INL, to an impression that utility surveying, and by inference shallow geophysics, can't supply the answers being sought in the day to day operations occurring at the site.

This lowest level designated Tier 1 can also be called 'standard locating'. It is the typical product supplied for the most part throughout the industry and consists of "tape and paint" surveys employing radio frequency (RF) detectors with some two-dimensional ground penetrating radar (GPR) profiling. Processing and imaging of the GPR data is usually confined to hyperbola picking of the two dimensional GPR profiles and the attempted correlation of features between the spatially isolated profiles. Many times the final product is just the painted lines with no report or maps to refer to. Due to the low level of 
data density that causes an inability to correctly correlate features in the ground, and the lack of precision in the data positioning for the accurate transfer of the interpretation to maps, this level of surveying fails to provide the product, geophysical or informational, currently being sought at the INL. Simply put the surveys don't collect enough data to correctly image the subsurface utility structures, the position of the located utility is incorrect, and when the paint on the ground is no longer visible the survey must be, and often is, repeated. The problems common to Tier 1 surveys are data density, positional accuracy and precision, as well as a result with no inherent institutional memory. These are addressed in a Tier 2 level survey.

This next level of product, the Tier 2 survey, has been called 'enhanced surveys' at the INL. This level of effort typically uses, in addition to the standard RF locating instruments, a suite of modern geophysical tools, such as single and multi channel cart mounted GPR, magnetic sensors and electromagnetic induction tools such as the EM61. The main differences between Tier 1 and Tier 2 are the data density, the precision in data positioning, and the change in data imaging and interpretation efforts of the GPR data blocks. While many standard surveys have historically used single or widely spaced GPR profiles, a Tier 2 effort uses a dense closely spaces collection of profiles, usually spaced one half to one antenna width, with data collected in orthogonal directions. Collecting data in two directions is essential as utilities that are parallel to the GPR profile orientation tend not to be imaged very well.

The integration of a precise positioning system improves the survey and the results in several ways. First, it allows for the economic collection of a dense data set by cutting the field collection time by a factor of two (no more measuring tapes to lay out). Next the positioning system, when tied in with the local survey grid, provides for precise repeatable positioning regardless of subsequent site operations that may remove surrounding structural references. The collection of data in a common local coordinate system also aids in the transfer of results to existing maps. Modern geophysical equipments, tied to the positioning system, provide an integrated data stream that can be fed, after some processing, into the local data management system for later access. This high data density and accurate positioning allows for more thorough and correct imaging and interpretation of the complex structures by the geophysicist.

Interpretation through time slice animation allows the interpreter to distinguish the real response from a utility feature (clearly migrating behavior of the hyperbola) from a false positive due to striping or mechanical issues. In addition subtle features such as old trench lines, old excavations and smaller radius utilities can be discerned where as Tier 1 survey results tend to not be useful. One evident observation is that a Tier 2 survey has not greatly advanced geophysical technology; it has just used existing technology in a better way. This is true and a boon to the geophysicist as a Tier 2 surveys does not necessarily require expensive re-tooling.

Tier 3 technologies can be seen as the cutting edge in utility designation (Birken et al., 2002). This level takes the Tier 2 concepts, density-positioning-imaging, and adds two other concepts.

- A rapid data collection system, usually vehicle mounted or towed, with multiple, coupled, sensor platforms.

- Advanced processing routines to clarify the image and interpretation process.

While Tier 3 technology/product provides superior results, they currently suffer from problems with respect to the average utility projects. First these surveys generally require large open areas with easy vehicle access. This makes it extremely useful for the large survey scale (city blocks). However it can be cost prohibitive for the typical small to medium scale utility surveys or for surveys where obstructions will prevent full coverage. The main difference between Tier 2 and Tier 3 is that of scale, both in area of coverage and in later processing and interpretation. For a variety of reasons common to others performing this work, such as scale and budget, INL has not yet employed Tier 3 surveying. 


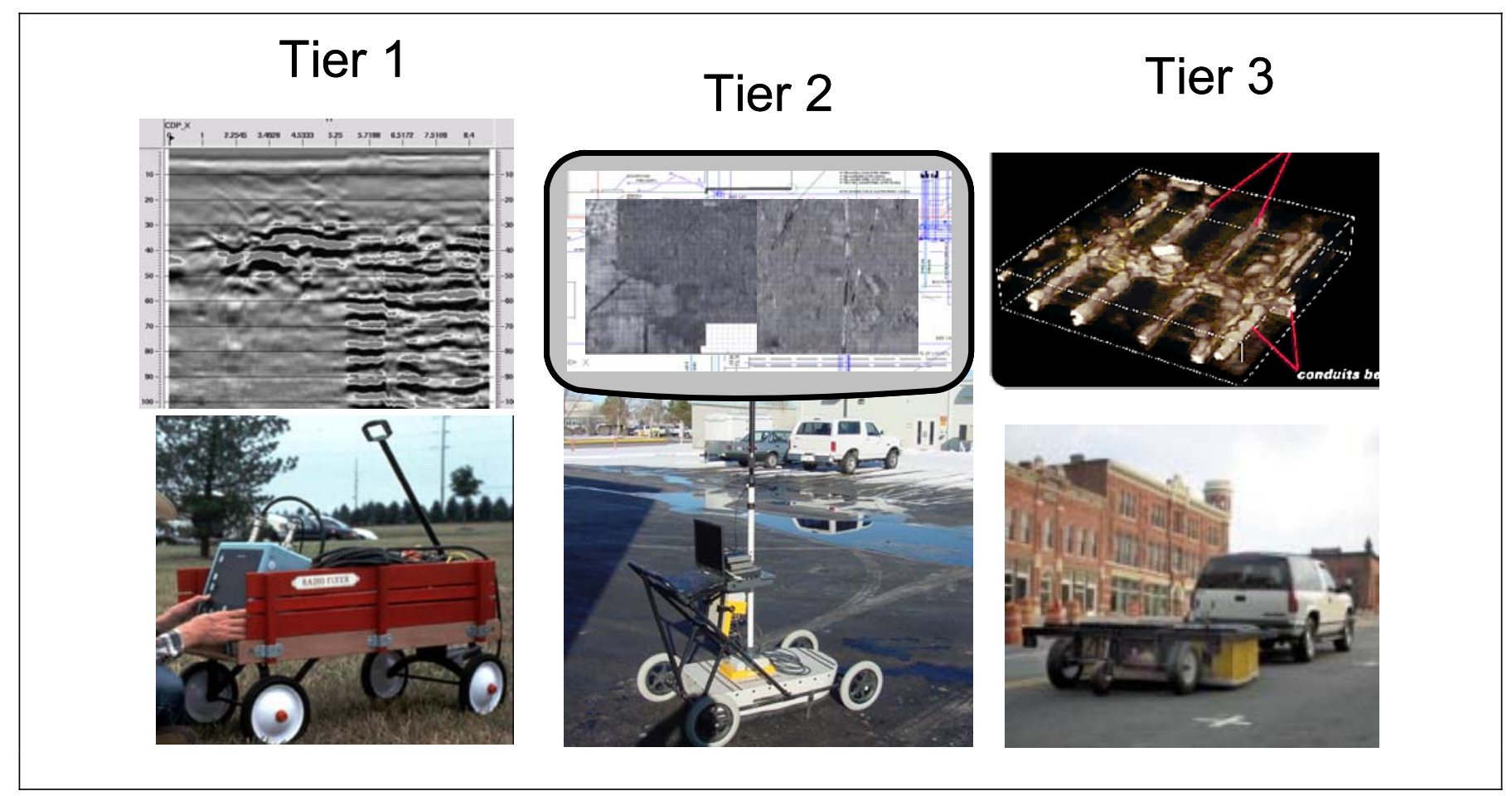

Figure 1: Basic description of Technology/Product Tiers in geophysics applied to utility issues. The images under Tier 1 reflect an older technology and processing that uses interpretation based on hyperbola recognition in isolated vertical GPR sections to define possible utilities. The center images under Tier 2 shows a more advanced technology with an integrated laser positioning system attached to the GPR and the time slice interpretation above. Typical Tier 3 results are shown at right with an example of sensor deployment below. The Tier 3 example is pulled from the Witten Technologies web site.

\section{INL Issues}

While organizational issues, basically available information, is of concern throughout the utility world, additional issues, due to the legacy nuclear work, face locating and identification efforts at DOE institutions. Of concern for typical utility identification purposes is the lack of access to utility end points at the INL. In many urban and civilian settings utilities come to the surface external to a building (usually for metering purposes) and aid in identification of the specific utility type (e.g. water-blue, gasyellow, or power-red). However, at the INL site almost all utilities penetrate the buildings below grade and into areas that are difficult if not impossible to access. In addition to the lack of access is the possibility that the unmapped legacy utilities, pipes, conduits and the areas where these have been exposed are highly contaminated with radiological and other hazardous materials. This makes unique identification of utilities a hazardous and potentially liable proposition and the possibility of legal ramification has caused us to stop identifying utilities by type. Essential we identify all utilities designated at the INL as of an unknown type (pink).

From experience working with the maps and information available at legacy sites, it is obvious that the maps generally reflect utilities installed at depth (greater than two to three meters) for large projects that included the costs for adding the as-built information to the relevant site map. Smaller projects tended to install utilities at depths less than two meters and for whatever reason, the final markups have rarely made it on to the maps. This has two implications. First the zone of missing 
information (upper two meters) roughly corresponds to the depths of penetration for the geophysical instrumentation making geophysical investigation very relevant to the utility issue. Second the product produced by geophysical mapping (inferred utility positions) rarely conforms to what is present in the existing drawings (utilities deeper than two meters). Unless this is made clear to the customer, they might assume the geophysics didn't adequately answer their needs and they may ignore the results.

Finally an issue that has driven utility mapping at INL away from the paint-on-the-ground product to an integrated map product is the issue of work scheduling. Often at the INL the work dependent on the utility locating effort has proceeded three to six months following the locating effort. This has led to added project costs from repeating utility surveys, occasionally as many as three to four times.

\section{INL Approach}

With the lack of adequate information and the heightened risk involved with hitting contaminated utilities at the INL, we have been working towards improving the utility locating results above the paint-on-the-ground level of product (Tier 1). By incorporating lessons learned from the Sterling report as well as the advances made by the people working at the Tier 3 level, we set out to increase the product level delivered to site personnel to Tier 2. This effort has included increasing data density with the available instruments and tying these instruments to a robust positioning system, currently an ArcSecond laser positioning system. In addition several in-house codes were developed to display the data and to create animations of time sliced data for interpretation. This effort has greatly increased the information available to site personnel when they plan and excavate in areas where this approach has been applied. Following are several examples of the work being produced at the INL site including a comparison between a Tier 1 survey and an early Tier 2 survey and an example of results from a current Tier 2 survey.

\section{Examples}

\section{Hot Cell Line Comparison and lessons}

Following the effort to understanding the problems facing INL in its utility locating work, an example survey was performed to illustrate the increased information and product quality in a Tier 2 survey over a Tier 1 survey (Scott, 2002). This was performed for a small survey attempting to identify a radiologically hot drain line leading from an experimental building on site (Figure 2). The issue was whether the target line ran next to or under the foundation of a nearby office building (green lines) requiring a substantially higher cost in retrieving the line in the latter case. In the Tier 1 approach an external company was brought in to perform a standard survey with a $250 \mathrm{MHz}$ GPR. Profiles were collected with a line spacing of 1 meter which was actually tighter than a typical Tier 1 survey. The result from the interpretive approach (2-D hyperbola picking) yielded a possible six lines in the target area with two corresponding to the two suspect line locations.

Following this a Tier 2 survey was performed using dense GPR (250 MHz) profiling in two orthogonal directions. Differences in the data collection and processing are shown in Table 2. For the Tier 2 survey a Mala GPR cart had been modified to mount a global positioning system (GPS) antenna and a local GPS base station was setup to provide differential corrections to meet requested centimeter accuracy. This approach for positioning failed to provide adequate data during the entire survey due to building interference causing multi-path problems and lost signals (blocked satellites). Even with the loss of consistent precise positioning data from the GPS, the GPR data was processed and imaged in a time 
slice aspect yielding superior information concerning the target drain line and other utilities in the area (Figure 3). As seen in this figure, the data presented in this manner allows not only for the identification of the hot cell line (\#1) but also indicates areas of concern where excavation might encounter other utilities (\#2 and \#3). The advantages of being able to recognize what we have termed 'Areas of Concern', in safety and excavation procedures, far outweigh the awkwardness in admitting that we don't know exactly what the data is telling us in this area. This added information greatly aided the planning and eventual removal of the identified drain line.

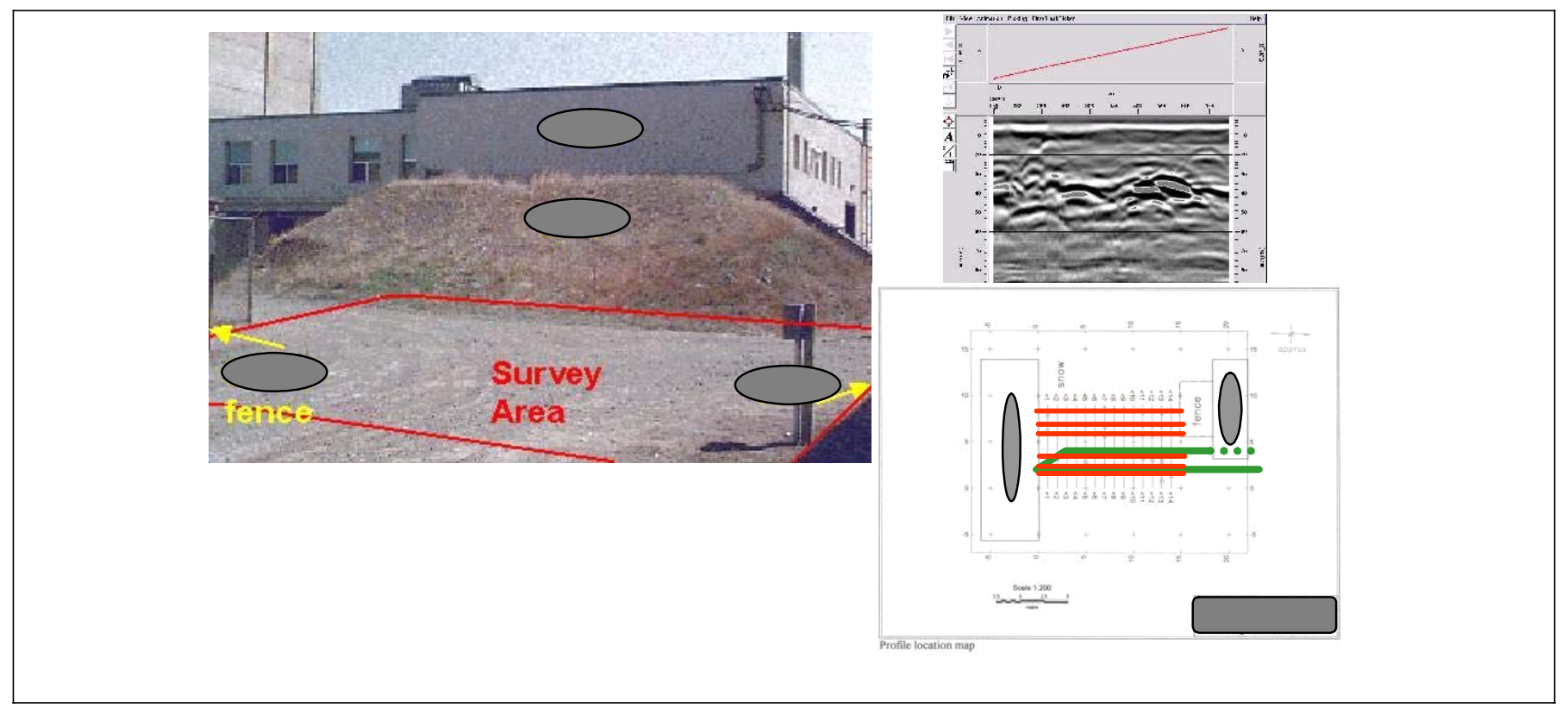

Figure 2: An image of the survey area and results from the Tier 1 survey. As seen in the photo the surface was a smooth gravel covered area positioned between buildings with the drain coming from a building on the right (not shown). The line was found near the top of the survey area at the foot of the earthen mound (shielding). Several Radiological Buffer areas border the survey area on the right and prevented the survey from running right up to the source building. The profile data on the right is typical of this survey and the interpretation below it was produced from recognizing and placing hyperbolas on a map. These hyperbola 'hits' are then correlated to produce the six red interpreted utilities on the map. The green lines represent a priori knowledge about the possible position of the targeted drain line. It's rather obvious that the Tier 1 effort produced a result whose usefulness is suspect. Building numbers have been covered at the clients request and the boilerplate for the company responsible for this work has been covered as well since it's not really their fault that Tier 1 results tend to be relatively poor. 
Table 2: Data Acquisition/Processing Parameters - Hot Cell Drain Line

\begin{tabular}{|l|l|}
\hline \multicolumn{1}{|c|}{ Tier 1 } & \multicolumn{1}{c|}{ Tier 2 } \\
\hline - $250 \mathrm{Mhz}$ GPR & $\bullet 250 \mathrm{Mhz}$ GPR \\
- Antenna dragged & $\bullet$ Cart based \\
- Time based triggering & $\bullet$ Distance based triggering $(2 \mathrm{~cm})$ \\
- Cloth tape positioning & $\bullet$ GPS differential positioning \\
- 1 meter spacing & $\bullet 1 / 3$ meter line spacing \\
- East-West Profiles only & $\bullet$ East-West and North-South profiles \\
- Interpreted using hyperbola picking and & $\bullet$ Interpreted using time-slice animation \\
\hline
\end{tabular}

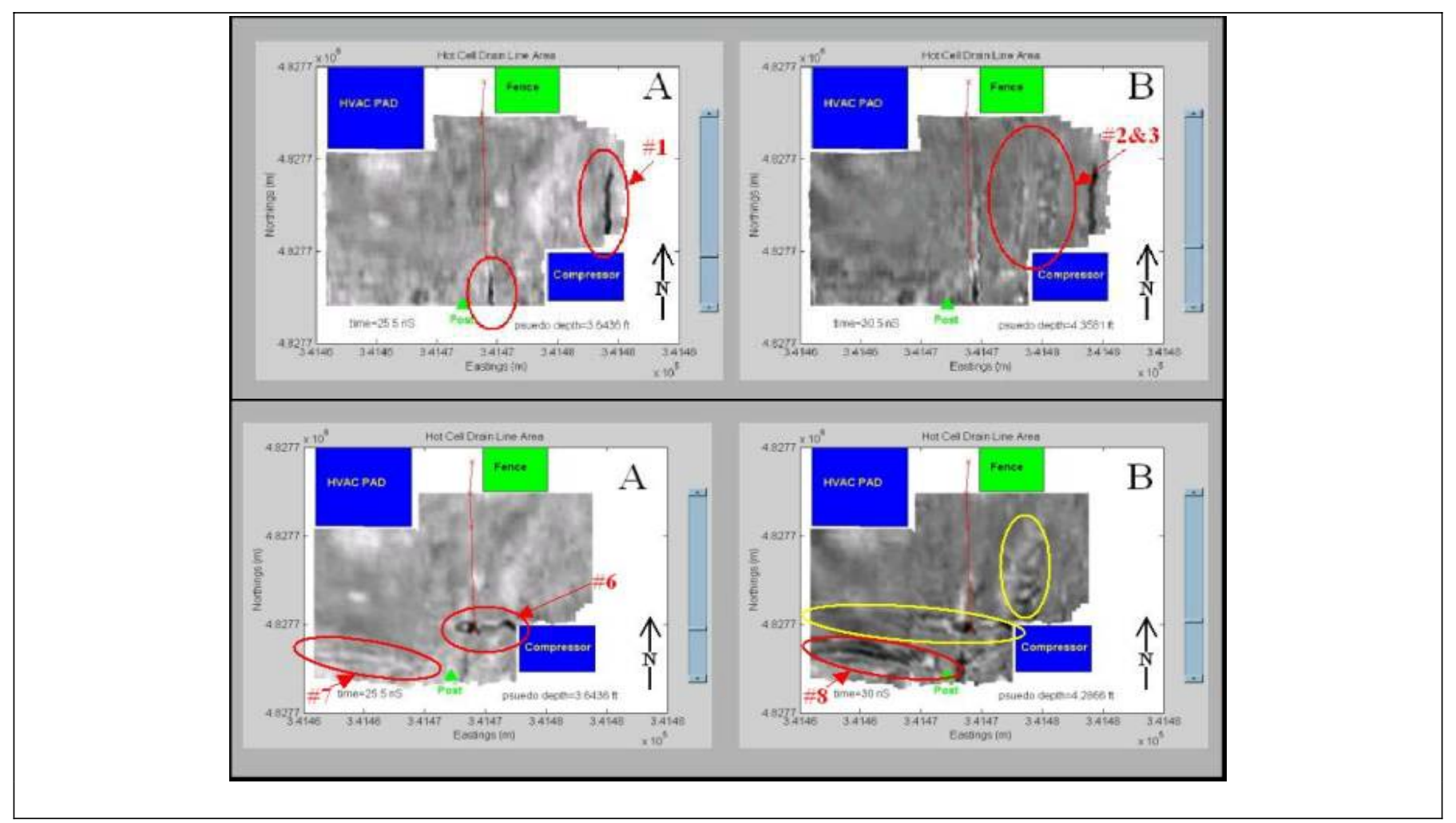

Figure 3: Results from a Tier 2 survey in roughly the same area as the Tier 1 effort in Figure 2. Orientation has been rotated 90 degrees from the earlier result. These time sliced images were pulled from the animation used to interpret the complete subsurface utility structures. They are typical of the results from this effort and show quite a bit more information than the six possible lines from the earlier Tier 1 survey. The top images are two slices from the East-West oriented profiles and show features with a predominantly North-South orientation. The bottom images are time slices from the North-South oriented profiles and will show features which tend East-West. The feature labeled as \#1 is identified as the target drain line while other nearby utilities are also indicated. Features labeled as \#2 and \#3 are visible in both orientations. While it is unclear exactly what's occurring in this area, the features are clearly a cause for concern and have come to be called 'areas of concern.' In total more than 10 features were uniquely identified with only one corresponding to the line found by radio frequency locating (thin red line with red x's trending N-S in center of images). 
Following the efforts and lessons learned from the hot cell drain line, improvements were made to the process including the integration of an Arc Second laser positioning system (see center bottom photo in Figure 1). This removed the problems associated with GPS in and among buildings and provides excellent resolution in positioning (generally sub-centimeter). The laser positioning system is useful both when integrated with the geophysical equipment as well as a stand alone survey instrument. When used alone the system is useful in setting up well defined survey areas and recording utility position data found with radio frequency locators in the same coordinate system as the other geophysical data. A procedure has developed to allow for economical collection of data which minimizes set up time and redundancies in the process.

- Use radio frequency locators to find obvious utilities. Paint results on ground

- Setup laser positioning system in static surveying mode to define survey boundaries and record positions found with the earlier radio locating

- Place laser system on geophysical instruments in roving mode and collect necessary geophysical mapping data (GPR, EM61, Magnetic Gradiometry).

- Process and interpret geophysical mapping data

- Setup laser surveying system a second time in static mode and paint results on ground.

- Place all results on map.

This procedure allows for easy positioning as both times the positioning system is set up the same way (same local coordinate system). The continuity of the positioning system also allows all data sets to be recorded in the same coordinate system thus allowing easy integration of data and maps. Depending on the size of the area to be surveyed, we've estimated this procedure represents a time cost of roughly one and a half times the cost of a Tier 1 survey. Given the increase in information versus the risk associated with excavation we've found our site customers more than willing to pay the extra costs.

\section{Current Tier 2 example}

A chance to further demonstrate the advantages of Tier 2 surveying arrived with the request to survey a large area planned for a series of deep excavations (three to four meters). This survey within the Reactor Technology Complex (RTC) at the INL comprised four areas each approximately thirty to sixty meters on a side. As part of the Tier 2 approach multiple instruments were employed including a modern digital RF locator, a Mala cart mounted GPR and an EM61 Mk2. The EM61 is an electromagnetic induction co-axial loop antenna system ideal for locating metal and tends to have greater penetration than the GPR. The ArcSecond laser positioning system was used to survey and was integrated with the GPR and EM61.

Data and results from one area in this recent survey are shown in Figures 4 through 6 . Figure 4 displays an example of typical GPR time slice data pulled out of the time slice animation. This data presents the East-West oriented profiles from an area approximately sixty meters by thirty meters. The GPR data was collected in orthogonal directions in two overlapping sections, roughly thirty meters square with a five meter overlap between sections. This subdivision of the area, along with an increase to half meter line spacing (no antenna overlap) was made to allow for smaller datasets and to ease interpretation. In similar surveys we have found it difficult to adequately interpret the time slice animations in datasets greater than about fifteen to twenty meters on a side. Here interpretations were made on the original thirty meter square data set and on subsets of the data roughly fifteen to twenty meters on a side. These GPR results found some utilities that seem to correspond to major utility corridors shown in the original utility map but the limit of penetration here was approximately 2 meters and many of the utilities inferred from this data were not present on the supplied map (Figure 6). 
Figure 5 shows the EM61 data for the same area. The EM61 was used in this survey to locate deeper underground features. In addition to indications of the common utility corridors seen in the GPR data, the EM61 data in this area was instrumental in locating a hidden underground tunnel that was roughly 3 meters deep, too deep for the GPR. In other areas the EM61 located a series of deep structures associated with an abandoned cathodic protection system.

Results from the different methods were combined and placed as new layers on the existing AutoCad maps (Figure 6). These results show a common problem found at these sites. While the maps show a well defined utility structure, most of the utilities plotted are at depths of 3 meters or more. Very little of the utilities in the upper 2 meters are represented in current drawings. In this sense the Tier 2 geophysical results are useful and in fact are required to show the utility structure in the upper 2 meters that will be encountered when excavating to the deeper levels.



Figure 4: GPR time slice data from a recent utility project. This data represents an area approximately 60 meters by 30 meters. The GPR data was collected in two overlapping sections ( 5 meter overlap) to allow for smaller data sizes and to ease interpretation. Interpretation is problematic for data sets greater than about 15 to 20 meters on a side and these data sets were further divided for interpretation. This study found some utilities that seem to correspond to major utility corridors in the map, but many of the utilities inferred from the geophysical data are shallower than those shown on the map (Figure 6). 


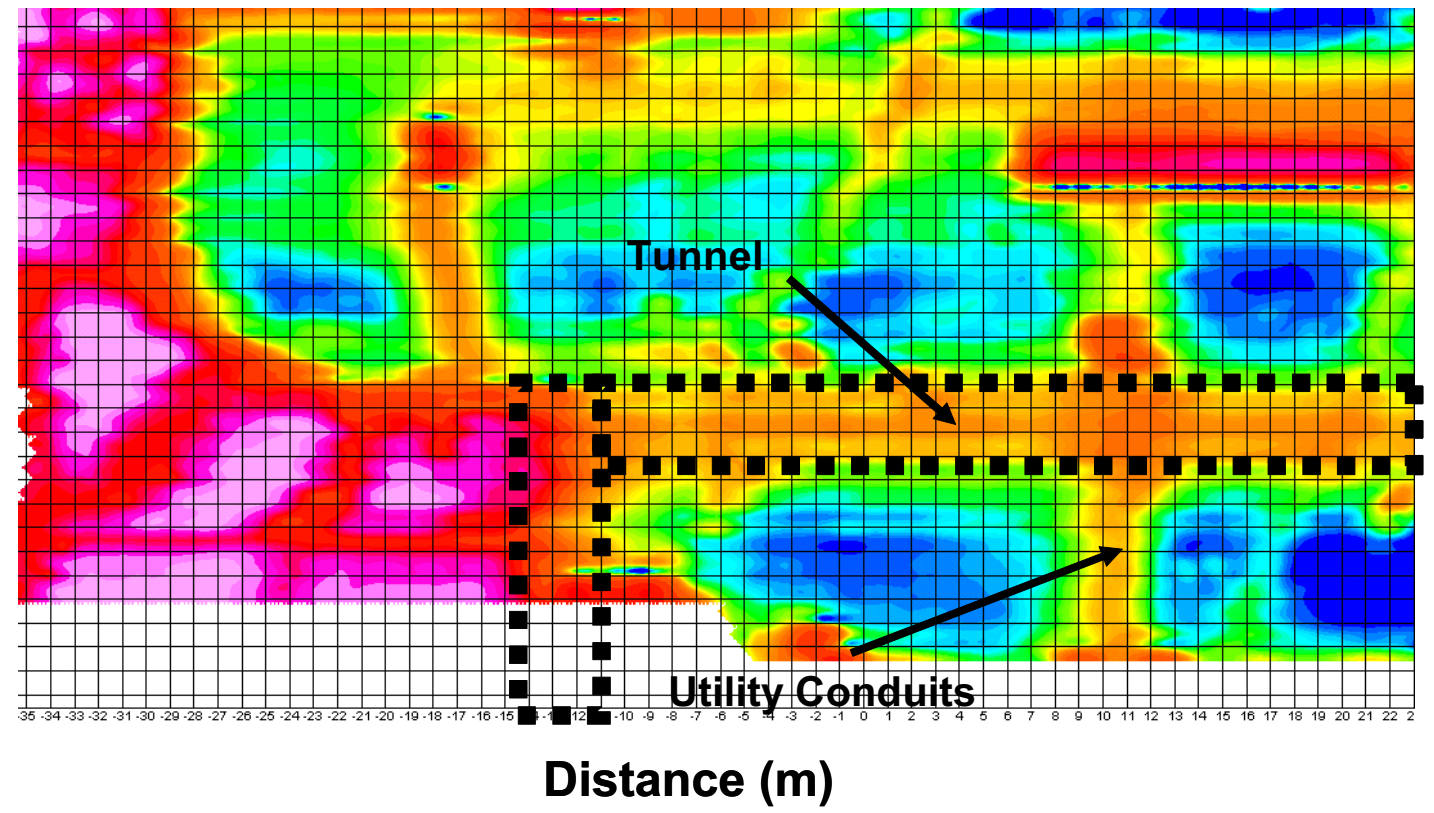

Figure 5: In addition to GPR, the EM61 Mk2 induction instrument was used in this survey to locate major underground features that may have been too deep for the GPR. This EM induction data corresponds in position to the GPR data in Figure 4. In addition to common utility corridors seen in both datasets, the EM61 data located an underground tunnel that was too deep for imaging with the GPR. 


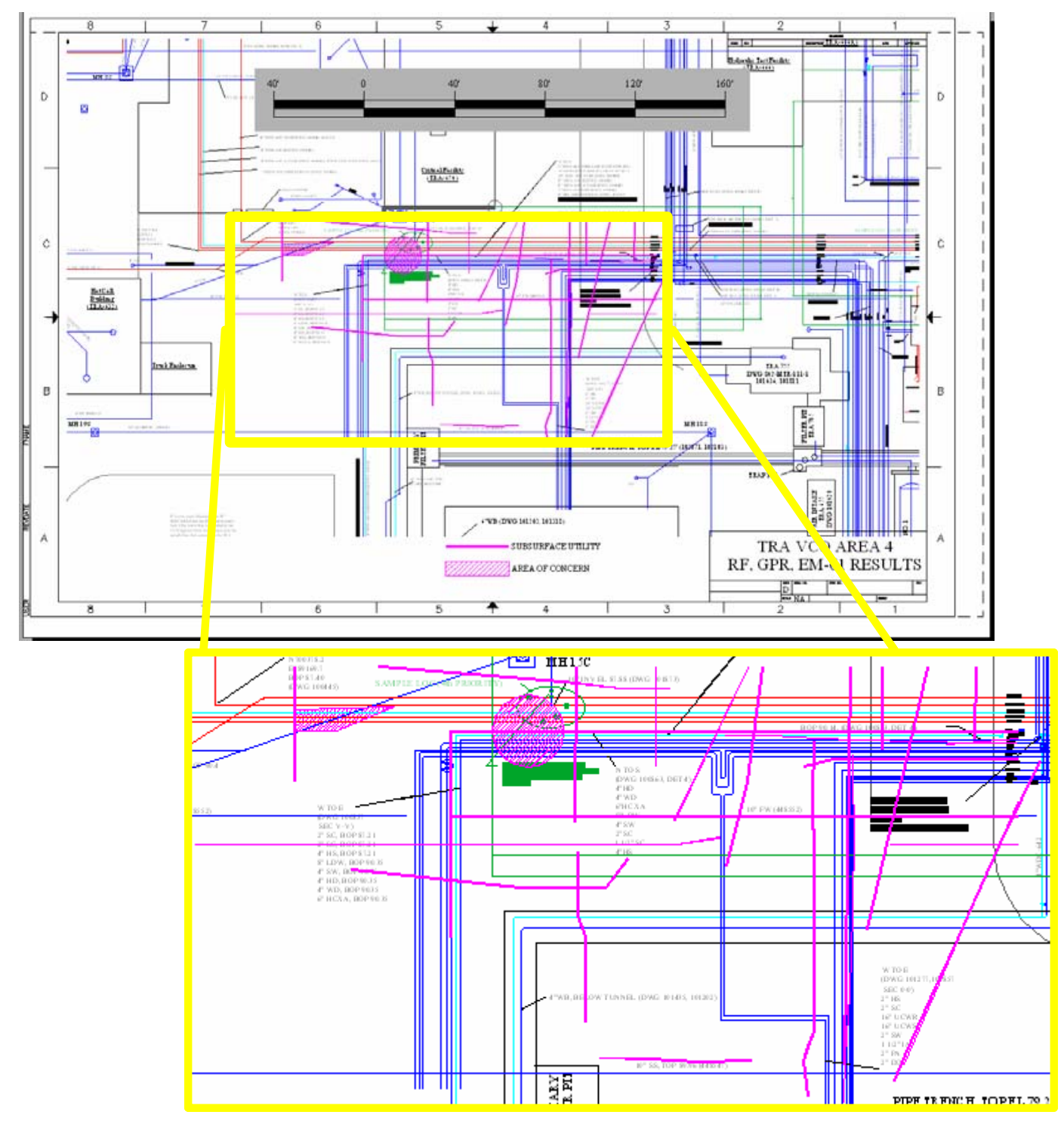

Figure 6: An example of the final utility designation product being supplied at the INL. The engineering Autocad map supplied by the customer is modified with layers corresponding to interpreted (designated) utility locations. The zoomed section corresponds to the surveyed areas in Figures 4 and 5 and show the utilities located tend not to correspond to the deeper utilities that are on the map. Areas of concern are also indicated by hatch filled features and indicate areas where excavation should proceed carefully. 


\section{Discussion and Conclusion}

With the lack of adequate information and the heightened risk involved with hitting contaminated utilities at the INL, we have been working towards improving the utility locating product from paint-on-the-ground (Tier 1) to map referenced drawings delivered to site personnel (Tier 2). By incorporating external standards (ASCE) and working to internally generalized descriptions defining level of geophysical effort (Tiered system), we have been able to significantly increase the quality level of information delivered to the engineering and excavation personnel at the INL using standard available geophysical instruments.

A comparison study employing standard and enhanced surveys shows a substantial increase in information regarding the subsurface utility structure can be gained by performing a Tier 2 survey. By incorporating an accurate and easy to use data positioning system, and developing procedures to remove redundant actions, Tier 2 surveys can be performed with only small incremental costs to the project.

The combined approach of developing and following standards, and increasing the data (information) density have led to a significant decrease in utility hits on the projects that have employed the Tier 2 level of effort.

\section{References}

ASCE, 2003. Standard Guideline for the Collection and Depiction of Existing Subsurface Utility Data, ASCE Standard CI/ASCE 38-02, 2003

Birken, R. et al., 2002. Efficient large scale underground utility mapping with a multi-channel groundpenetrating imaging radar system, SAGEEP 2002. EEGS, Las Vegas, NV.

Scott, C., 2002, Buried Infrastructure Locating Study: TRA Hot Cell Drain Line, TRA 713 Tank Site, INEEL Internal Report INEEL/INT-02-01432, November 2002.

Sterling, R.L., 2000, Utility Locating Technologies: A Summary of responses to a Statement of Need Distributed by the Federal Laboratory Consortium for Technology Transfer, Federal Laboratory Consortium Special Reports Series No. 9, ISSN 1075-9492C, February 2000. 